\title{
O USO DE ANTIINFLAMATÓRIOS ESTEROIDAIS E NÃO ESTERIODAIS NO CONTROLE DA DOR E DO EDEMA EM CIRURGIA DE TERCEIROS MOLARES
}

\author{
Ricardo Natã Fonseca Silva*, Lúcia Coelho Garcia Pereira** \\ Autor correspondente: Ricardo Natã Fonseca Silva - ricardoodonto51@hotmail.com \\ * Mestrando em Patologia pela Universidade Federal de Goiás. Cirurgião Dentista - Centro Universitário de Anápolis \\ ** Doutora em Dentística - Faculdade de Odontologia de Bauru/Universidade de São Paulo. Professora do Centro \\ Universitário de Anápolis
}

\begin{abstract}
Resumo
Introdução: Terceiros molares são os últimos dentes a irromperem na cavidade oral, e em alguns casos sofrem agenesia, impaç̧ão ou permanecem inclusos. Durante a cirurgia de remoção, ocorrem injúrias aos tecidos orais, provocando sintomatologia dolorosa e edema. Para o combate destes, faz-se necessária a utilização de fármacos que controlam a inflamação, como os anti-inflamatórios não-esteroidais (AINES) e corticosteróides. Para se obter um melhor resultado, a combinação de AINES e anti-inflamatórios esteroidais pode ser utilizada em pacientes, tanto como medicação preemptiva, como pós-operatória. Objetivo: realizar uma revisão da literatura com o intuito de verificar quais classes de medicamentos apresentam melhores resultados pós-operatórios diante dos quadros de dor e edema em exodontias de terceiros molares. Materiais e métodos: Como técnica para a seleção dos trabalhos optou-se por incluir estudos presentes nas bases de dados Scielo, Lilacs e Pubmed publicados entre os anos de 2000 a 2014. Considerações Finais: O uso de corticostróides se faz de maneira preemptiva com o objetivo de reduzir o quadro de edema, enquanto os AINES tem maior utilização no pós-operatório, na tentativa de amenizar a sensação dolorosa.
\end{abstract}

Palavras-chaves: Terceiros molares; Dor; Edema. 


\title{
PAIN AND SWELLING CONTROL THROUGH NON- STEROIDAL ANTI-INFLAMMATORY DRUGS (NSAIDS) AND CORTICOSTEROIDS IN THIRD MOLARS SURGICAL EXTRACTION
}

\begin{abstract}
s
Introduction: Third molars are the last teeth to erupt through the oral cavity and in some cases they are congenitally missing, display impaction or remain included. There are many reasons for their removal: decay, lack of space in the dental arch or problems during its eruption. During surgical extraction, oral tissues may be injured, which causes painful symptoms and edema. To combat these surgical complications, it is necessary the use of drugs that fight inflammation, non-steroidal anti-inflammatory drug (NSAIDs) and corticosteroids. To obtain best results, the combination of NSAIDs and steroidal anti-inflammatory drugs are used in patients, either as preemptive medication and as postoperative medication. Objective: Performing a literature review in order to determine which drug classes have better postoperative outcomes for pain and swelling in of third molars extraction. Materials and methods: Studies present in SciELO, Lilacs and Pubmed databases published between 2000-2014 were included. Final considerations: Corticosteroids are used preemptively in order to reduce swelling frame, while NSAIDs have greater use in the postoperative period, in an attempt to reduce the pain.
\end{abstract}

Keywords: Third molars; Pain; Swelling.

\section{INTRODUÇÃO}

Quando os terceiros molares irrompem na cavidade oral, podem ou não erupcionar em alinhamento correto e funcional. (1) Isso se deve ao fato de os terceiros molares serem os últimos dentes a completarem sua formação e cronologicamente serem os últimos a irromperem, ficando susceptíveis à falta de espaço no arco dentário ou não conseguem romper o denso revestimento ósseo, ou o tecido mole sobreposto. ${ }^{(2)}$

Chaves Júnior et al. (1) afirmam que, quando inclusos, são potencialmente capazes de causar transtornos e prejuízos à saúde bucal do indivíduo, como doença periodontal, pericoronarite, reabsorção radicular dos dentes adjacentes e desenvolvimento de cistos e tumores, sendo a exodontia indicada.
A idade recomendada para exodontia de terceiros molares com menores índices de complicações e um melhor prognóstico é entre 17 e 18 anos. (3)

As complicações mais comuns das exodontias são: infecção, alveolite, disestesia, parestesia, hemorragia, dor e trismo. (3) Quanto aos acidentes transoperatórios mais frequentes são apontadas a fratura dento alveolar, injúria ao dente vizinho, fratura da mandíbula, dano a ATM, hemorragias, lesões nervosas, comunicação buco sinusal e lesões aos tecidos moles. $(4,5)$

O uso de analgésicos e anti-inflamatórios não esteroidais, analgésicos de ação central e esteroides têm sido empregado para o controle da dor e edema no pós-operatório. ${ }^{(6)}$

O edema cirúrgico alcança sua expressão máxima em 48 a 72 horas após o procedimento, começando 
a regredir por volta do terceiro dia e tem sua resolução normalmente em um período de 7 dias, afirma Poeschl et al.(7) O uso de anti-inflamatórios esteroidais no pré-operatório reduz significativamente o edema. ${ }^{(8)}$

Já a dor pós-operatória atinge seu pico em torno de 12 horas após o fim da exodontia, diminuindo rapidamente após este período. Em situações de normalidade, o quadro doloroso desaparece em torno de dois dias após o procedimento cirúrgico. ${ }^{(2)}$

O objetivo do presente trabalho é verificar quais classes de medicamentos apresentam melhores resultados pós-operatórios diante dos quadros de dor e edema em exodontias de terceiros molares.

\section{MÉTODOS}

Como técnica de seleção dos trabalhos optou-se por incluir estudos presentes nas bases de dados Scielo, Lilacs e Pubmed. Os critérios de inclusão estabelecidos foram: artigos originais disponibilizados na íntegra e na forma online no idioma português ou inglês, capítulos de livros e dissertações ou teses publicadas no período compreendido entre os anos de 2000 e 2014. Como critérios de exclusão foram estabelecidos artigos de pesquisa bibliográfica e de reflexão e artigos repetidos em diferentes bases de dados.

Após seleção dos artigos relacionados ao tema proposto, fizemos uma revisão de literatura, com intuito de comprovarmos os fármacos que surtem resultados pós-operatórios mais positivos diante de dor e edema em cirurgias de terceiros molares.

Foram selecionados apenas os trabalhos que continham as seguintes palavras chave, anti-inflamatórios, edema, dor e terceiros molares, que são referentes ao tema "O uso de anti-inflamatórios esteroidais e não-esteroidais no controle da dor e do edema em cirurgia de terceiros molares".

Após a seleção prévia destes trabalhos, esses foram reavaliados pelos autores desse estudo, para seleção final dos mesmos e elaboração da Revisão de Literatura

\section{REVISÃO DE LITERATURA}

\subsection{TERCEIROS MOLARES}

Segundo Hupp et al(2) os terceiros molares, são os últimos dentes a erupcionar na cavidade oral, reduzindo a possibilidade de encontrar espaço nos maxilares para irromperem, podendo então ficar impactados.

Segundo Marzola(9) a impacção dos terceiros molares pode aumentar o risco de manifestação de lesões, como mobilidade dentária, reabsorção radicular de dentes vizinhos e tumores odontogênicos, em virtude de que, os dentes em questão estejam aplicando alguma força, coronária e/ou radicular, sobre os segundos molares, promovendo uma reabsorção óssea na crista alveolar tendo como resultado negativo a mobilidade dentária. Quanto aos tumores odontogênicos, podem ocorrer ao redor da coroa de um dente impactado, normalmente inferior. ${ }^{(10)}$

O avanço de técnicas radiográficas, como a radiografia panorâmica, tomografia computadorizada e técnicas de localização radiográficas como Parma e Donovan, permitem a previsão precoce da retenção destes dentes e sua extração tornou-se um procedimento comum e rotineiro para o cirurgião buco maxilo facial.(11)

Existem fatores como ausência de espaço na arcada dentária, indicação ortodôntica, impacção, presença de tumores circundando a coroa dos dentes em questão e destruição coronária, que levam à necessidade de exodontia. Para o planejamento da cirurgia é necessário a identificação do posicionamento dentário, previsão de osteotomia e avaliação da necessidade ou não de seccionamento do elemento dentário, visando intervenção menos traumática e prevenção de acidentes e complicações trans e pós-operatórias como hemorragias, traumas, comprometimento de estruturas nervosas, fraturas radiculares e ósseas, danos aos dentes vizinhos, dor, edema e infecção. (3) No estudo radiográfico as impacções dentárias, são classificadas 
segundo a angulação (mesioangular, horizontal, vertical e distoangular), em relação a borda anterior do ramo mandibular (Classe 1, 2 e 3 ) e em relação ao plano oclusal (Classe A, B e C), facilitando o estabelecimento de técnicas e recursos para as extrações. ${ }^{(11)}$

O procedimento de exodontia de terceiros molares frequentemente resulta em dor pós-operatória, edema e limitação da abertura bucal devido ao trauma cirúrgico, sendo que a cicloxigenase e as prostaglandinas desempenham função primordial nesse processo. Uma boa técnica cirúrgica e a manipulação cuidadosa dos tecidos minimizam a inflamação pós-operatória, mas não a previne. Fatores tais como idade, gênero, história médica, uso de métodos anticoncepcionais, presença de pericoronarite, má higiene oral, tabagismo, tipo de inclusão, duração do procedimento, técnica cirúrgica, experiência do cirurgião, uso de antibióticos no perioperatório ou de medicamentos intra-alveolares podem influenciar significativamente a dor e a inflamação desse tipo de intervenção. ${ }^{(12)}$

\subsection{DOR PÓS-OPERATÓRIA}

Peixoto et al. ${ }^{(13)}$ e Teixeira(14) definem dor como uma experiência sensorial e emocional desagradável, sendo considerada apenas como um sintoma, que irá orientar o diagnóstico das morbidades do indivíduo.

A Organização Mundial de Saúde (OMS) escalona a dor de forma uni e multidimensional. A escala unidimensional verbal, utiliza expressões para analisar o nível da dor, sendo elas: nenhuma dor, dor leve, dor moderada, dor forte e dor insuportável. $\mathrm{Na}$ escala numérica é utilizado uma escala de o a 10, sendo zero nenhuma dor e 10 maior dor possível. ${ }^{(15)}$

Além da avaliação do nível de dor, estabelecido pela escala numérica, a OMS também preconiza a escala de faces, que proporciona uma visão mais ampla, a partir do momento em que você consegue ver o paciente e estabelecer um grau de dor, olhando para este e comparando com o que nos foi proposto. Seguindo isto, temos a face dividida em cinco categorias de dor: ausência de dor, dor leve, dor moderada, dor forte e dor insuportável. ${ }^{(15)}$

Já a escala multidimensional, avalia aspectos psicofísicos como: aspectos sensoriais, afetivos, avaliativos e intensidade da dor. Aspectos sensoriais são propriedades mecânicas, técnicas de vividez e espaciais de dor; Aspectos afetivos: tensão, medo e respostas neurodegenerativas; Aspectos avaliativos: avaliação global da experiência dolorosa; Aspectos de intensidade de dor: expressões faciais como olhos cerrados, sobrancelhas franzidas, língua tensa e côncava. ${ }^{(15)}$

De acordo com Wannmacher et al ${ }^{(16)}$ a caracterização da dor é importante para a seleção da conduta analgésica a ser adotada. Sendo assim, classifica-se a dor segundo critérios temporais (aguda e crônica), topográficos (localizada e generalizada, tegumentar e visceral), fisiopatológica (orgânica e psicogênica) e de intensidade (leve, moderada e intensa). ${ }^{(17)}$

A intensidade da dor após a exodontia de terceiros molares impactados pode ser influenciada por fatores como, o uso de diferentes sais anestésicos antes do procedimento, sendo que anestésicos com uma maior estabilidade em suas ligações possuem um maior tempo de duração, e a utilização de lasers de baixa potência. ${ }^{(18)}$ Negreiros $^{(19)}$ e Aguiar et al, ${ }^{(20)}$ afirmam que o aumento da dor pós-operatória pode também ser em virtude da expansão de tecido causado pelo trauma cirúrgico, onde há uma intensa liberação de mediadores químicos responsáveis pelo processo inflamatório.

\subsection{EDEMA PÓS-OPERATÓRIO}

Edema é a expressão de exsudato ou transudação, e em uma cirurgia, provavelmente ambos os eventos ocorrem. ${ }^{(21)}$

O edema no pós-operatório contribui muito para o aumento da dor, pois há um crescimento da tensão nos tecidos, ocorrendo principalmente por volta do segundo e terceiro dia, após um trauma cirúrgico. ${ }^{(21)}$ 
Os principais fatores que contribuem para o edema são a duração da cirurgia e a dificuldade cirúrgica, podendo também estar relacionado com o grau de impacção. Diante da classificação de Pell e Gregory, os molares na posição III e C, apresentam um aumento na dificuldade cirúrgica e por isso um edema mais prevalente. ${ }^{(20)}$

Figura 1 - Classificação da posição dos terceiros molares segundo Pell e Gregory ${ }^{(22)}$

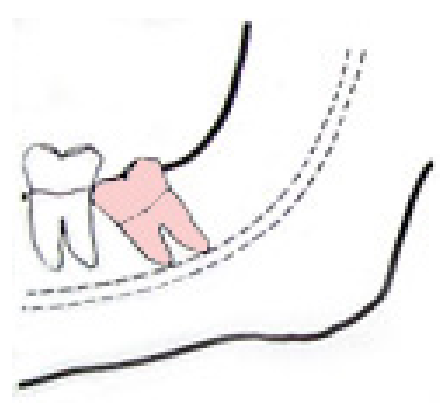

Class I

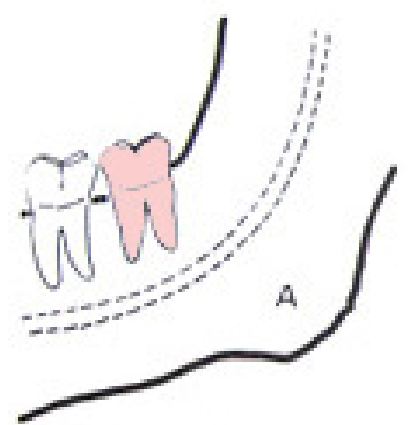

Position A

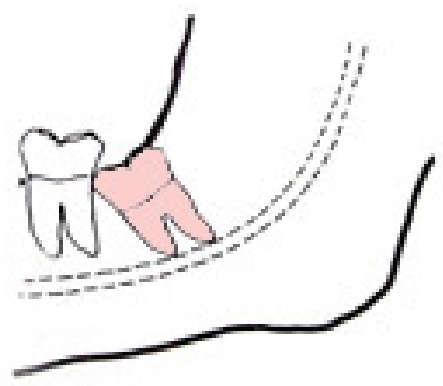

Class II

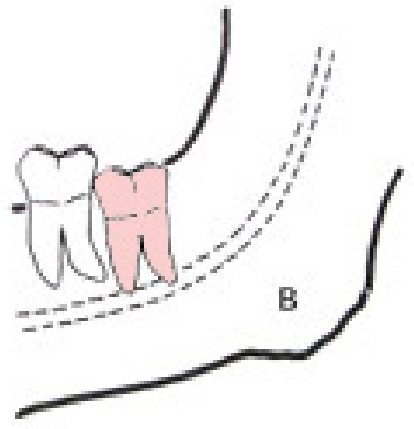

Position B

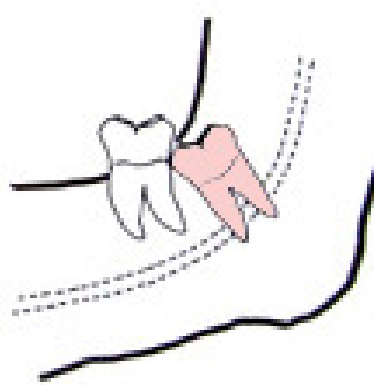

Class III

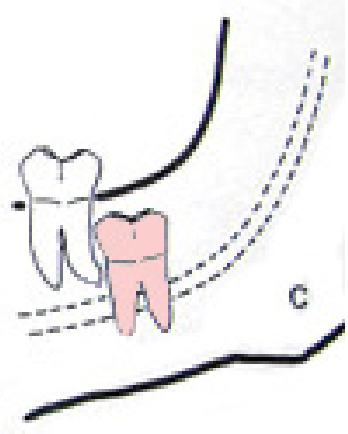

Position C

Fonte: Adaptada de Pell e Gregory.

Existem fatores, que influenciam em uma maior ou menor prevalência de edema, como sexo, idade, grau de dificuldade cirúrgica, experiência do cirurgião, o tamanho da incisão e a manipulação dos tecidos. ${ }^{(20)}$

Para uma melhor avaliação, existem métodos para medição do edema. Silva, ${ }^{(23)}$ descreve que um dos métodos mais utilizados é o de Markovick e Todorovic, consistindo na mensuração da ponta do mento até o bordo inferior do lobo auricular, sendo realizada no pré e pós-operatório para uma posterior comparação.

\subsection{CORTICOSTEROIDES NO CONTROLE DA DOR E EDEMA}

Drogas anti-inflamatórias esteroidais são capazes de reduzir a dor e o edema pós-operatório depois da exodontia de terceiros molares impactados. O tratamento cirúrgico induz a uma complexa interação entre a inflamação local e respostas neuro-hormonais em nível microscópico manifestando os cinco sinais cardinais da inflamação: calor, rubor, edema, dor e perda de função. ${ }^{(24)}$

Peixoto et $a^{(13)}$ e Simone et al $^{(25)}$ afirmam que corticosteroides previnem hiperalgesia através da inibição da fosfolipase A2 e inibem a liberação da 
cicloxigenase. Os mesmos atuam inibindo reações em cadeia que degradam os fosfolipídios das membranas celulares lesionadas responsáveis pela resposta inflamatória, portanto quanto menor a concentração destes mediadores nos tecidos, mais fraca é a resposta inflamatória. Desse modo, corticosteroides tornam-se a droga de opção mais aceita, usada tanto antes quanto após o procedimento cirúrgico de remoção de terceiros molares. ${ }^{(25)}$ Murugesan et al ${ }^{(26)}$ perceberam ainda que, os corticosteroides afetam o movimento, ativação e função dos leucócitos e previnem a síntese de prostaglandinas por inibição da cascata do ácido araquidônico.

Em um estudo comparativo feito por Murugesan et al, ${ }^{(26)}$ entre corticoides (dexametasona) e enzimas (serratiopeptidase), o anti-inflamatório esteroidal apresentou resultado mais favorável em relação à enzima no controle da dor e do edema pós-operatório.

Segundo Simone et al ${ }^{(25)}$ quando o efeito analgésico preemptivo de corticosteroides e Anti-Inflamatórios Não Esteroides (AINEs) são comparados através de escalas médias de dor durante as primeiras 72 horas, observa-se que o grupo tratado com corticoides apresenta baixos valores na escala analógica de dor, enquanto o grupo tratado com AINEs e placebo obtiveram altos valores na escala visual analógica. Resultados mostraram que os corticosteroides são mais eficazes no controle da dor.

O melhor desempenho do corticosteroide comparado com o AINEs pode ser atribuído ao mecanismo de ação desta droga. Sua provável ação está na microvascularização e no efeito celular, impedindo também a degola endotelial de leucócitos e diapedese através da parede capilar. ${ }^{(27)}$

Em um estudo realizado por Leone et al(27) comparando o efeito do corticosteroide (metilprednisolona) com o anti-inflamatório não esteroidal (cetoprofeno) para alívio da dor, pôde notar que não houve diferenças significativas no alivio da dor após exodontia de terceiros molares. Entretanto, utilizando o corticosteroide como medicação preemptiva nota-se a inibição na liberação de me- diadores químicos (bradicininas e outros) que minimizam a sensação dolorosa pós-operatória.

Quanto à redução do edema, Kocer et al ${ }^{(28)}$ relatam a administração de injeção de dexametasona no músculo masseter obtendo um resultado satisfatório se comparado a outras vias de administração do fármaco, como por exemplo a via oral. Essa redução se dá em virtude do processo farmacodinâmico, onde na administração enteral o fármaco necessita passar por todo o trato gastrointestinal, em contrapartida, na via parenteral há a deposição do medicamento diretamente no local da inflamação. ${ }^{(29)}$

\subsection{ANTI-INFLAMATÓRIOS NÃO ESTEROIDAIS NO CONTROLE DA DOR E DO EDEMA}

A classe de fármacos denominados Anti-Inflamatórios Não Esteroides (AINEs) inclui diversos ácidos orgânicos independentes, que compartilham propriedades anti-inflamatórias, analgésicas e antipiréticas, sendo utilizados no tratamento da dor branda a moderada. ${ }^{(17)}$

Os AINEs inibem as cicloxigenase $1\left(\operatorname{COX}_{1}\right)$ e a cicloxigenase 2 ( $\operatorname{COX} 2$ ), isoenzimas da cicloxigenase, que assim agem como inibidores diretos da síntese de prostaglandina e tromboxano a partir do ácido araquidônico. COX 1 é a forma constitutiva da COX, encontrada em plaquetas, células endoteliais vasculares, estômago e rins, estando envolvida na proteção da parede do estômago (prostaglandina E2), agregação plaquetária (tromboxano A2) e função renal (prostaglandina I2). ${ }^{(30)} \mathrm{COX} 2$ é a forma induzida na presença de inflamação, mas pode também ser encontrada em tecidos cerebrais e renais, na ausência de inflamação. Os AINES individuais inibem a $C O X_{1}$ e $C_{2} O X_{2}$ em graus variados e, portanto, quando utilizados em doses equivalentes, os perfis de segurança destes fármacos variam individualmente, mas há pouca diferença de um para outro em termos de eficácia. ${ }^{(29)}$ Devido ao processo inflamatório, células de defesa são ativadas e sinalizam moléculas das células do ácido 
araquidônico. Durante esta ativação, os metabólitos do ácido araquidônico, prostaglandina 12 e prostaglandina E2 causam vasodilatação, e especificamente a PGE2, aumenta a sensibilidade para estímulos de dor podendo mediar a febre e potencializar o edema. ${ }^{(30)}$

AINEs administrados no pré-operatório previnem sensibilização periférica com diminuição na sensibilidade de nociceptores periféricos induzidos por mediadores inflamatórios, tal como prostaglandinas, liberados no local da injúria. (30) Em um estudo duplo-cego cruzado realizado por Calvo et $a^{(30)} \mathrm{em}$ que foram feitas exodontias de terceiros molares inferiores bilateralmente medicando os pacientes no pós-operatório com AINEs inibidores seletivos da COX-2 (etoricoxib 120mg) e AINEs convencionais (Ibuprofeno 6oomg), os resultados não apresentaram diferenças em relação ao controle da dor pós-operatória. ${ }^{(28)}$

Após a remoção cirúrgica de terceiros molares, o curso pós-operatório é caracterizado por dor e edema afetando vários graus na qualidade de vida do paciente. "Esta lesão de tecidos provoca a liberação da ciclooxigense-2, que induz a atividade de prostaglandinas que sensibilizam nociceptores periféricos e induzem sintomas da inflamação". (30)

\section{DISCUSSÃO}

O uso de anti-inflamatórios não esteroidais inibem as cicloxigenases, impedindo a liberação de prostaglandinas com isso o quadro inflamatório no local da injúria tecidual se faz de maneira mais branda. Os corticosteroides promovem a inibição da fosfolipase $A_{2}$ impedindo a liberação do ácido araquidônico seguido de seus metabólitos (prostaglandinas, prostaciclinas e tromboxano). ${ }^{(25)}$

Silva ${ }^{(15)}$ descreveu maneiras para diminuir os sinais e sintomas pós-operatórios, utilizando os corticoides, que reduzem de maneira considerável o edema, melhorando desse modo, o nível de satisfação e conforto do paciente. Já os AINEs em dosagens recomendadas, mostram efeitos similares no controle de edema e abertura bucal.
Saar et $a^{(24)}$ Murugesan et $a^{(26)}$ relatam que o uso de corticosteroides em exodontias de terceiros molares apresentam uma diferença significante na melhora da dor e do edema nos três primeiros dias. Após este período, o quadro de melhora é equivalente, ou seja, tanto com AINEs quanto com o próprio anti-inflamatório esteroidal.

Menezes e Cury ${ }^{(31)}$ mostraram que o uso de AlNEs, preferencialmente meloxican e nimesulida, apresentam um controle na dor e no edema pós-operatório. Estes sintomas também podem ser amenizados pela administração de ibuprofeno. ${ }^{(31)}$

Quanto ao efeito analgésico preemptivo de corticoides e AINEs a comparação é feita por meio de escalas médias de dor durante as primeiras 72 horas, observando que o grupo tratado com corticoides apresentou baixos valores na escala analógica de dor, enquanto o grupo tratado com AINEs e placebo obtiveram altos valores. Resultados mostraram que o corticoide é mais eficaz no controle da dor. O melhor desempenho do corticoide comparado com o AINEs pode ser atribuído ao mecanismo de ação desta droga. (31)

Diversos cirurgiões buco maxilo faciais fazem o uso de medicação preemptiva em cirurgias de terceiros molares na tentativa de minimizar principalmente o edema. Simone et al(25) e Leone, ${ }^{(27)} \mathrm{com}$ pararam o efeito pré-operatório da administração das classes de antiinflamatórios, confirmando que o corticosteroide se comparado ao AINEs mostra uma melhora na redução do quadro de edema.

Associações de fármacos também são bastante utilizadas (corticosteroides + AINEs, corticosteroides + analgésicos de ação periférica, corticosteroides + analgésicos de ação central). Kocer et al ${ }^{(28)}$ defendem a associação de fármacos, relatando que há uma melhora significativa se comparado à administração dos fármacos isoladamente.

$\mathrm{Na}$ vivência clínica do cirurgião buco maxilo facial tanto AINEs quanto corticoides são administrados rotineiramente, entretanto existem algumas limitações quanto ao uso de anti-inflamatórios não esteroidais, por exemplo em pacientes renais crônicos e com problemas gástricos, sendo necessário 
a prescrição de corticosteroides, mesmo que estes não sejam a primeira opção. ${ }^{(16)}$

\section{CONSIDERAÇÕES FINAIS}

De acordo com a literatura consultada foi possível constatar que o uso de corticoides se faz mais acentuadamente como medicação preemptiva na tentativa de reduzir o quadro de edema em exodontias de terceiros molares. Em relação ao uso de AINEs, há uma maior prevalência de sua prescrição no pós-operatório para promover uma modulação da sensação dolorosa.

\section{REFERÊNCIAS}

1. Chaves Junior AC, Pereira ACL, Fronza BR, Oliveira HTR, Chagas OL, Silva TSN. Técnica cirúrgica para a remoção de terceiros molares inferiores e a classificação de Pell - Gregory: um estudo relacional. Rev. cir. traumatol. bucomaxilo-fac. 2006;6(4):65-72.

2. Hupp JR. Princípio do tratamento de dentes impactados. In: Hupp JR, III EE, Tucker MR. Cirurgia oral e maxillofacial contemporãnea. $5^{a}$ Ed. Rio de Janeiro: Elsevier; 2009. p. 160-164.

3. Sato FR, Aspirino L, de Araújo DE, Moraes M. Short-term outcome of postoperative patient recovery perception after surgical removal of third molar. J. oral maxilofac. surg. 2009;67(5):1083-1091.

4. Mercier P, Precious D. Risks and benefits of removal of impacted third molars. A critical review of the literature. Int. j. oral maxilofac. surg. 1992;21(1):17-21.

5. Negreiros RM, Milani BS, Baver HC. Acidentes nas exodontias de terceiros molares. In: Callegari A, Marcelo MCS, Bombana AC, coordenadores. Atualização clínica em odontológica: clínica do dia-a-dia. São Paulo: Aretes Médicas; 2008. p. 370-383.

6. Taube S, Pironen I, Yliparavalniemi P. Heliumneon laser therapy in the prevention of postoperative swelling and pain after wisdom tooth extraction. Proc. finn. dent. soc. 1990; 86(1):23-7.

7. Poeschl PW, Eckel D, Poeschl E. Postoperative prophylactic antibiotic treatment in the third molar surgery - a necessity? J. oral maxilofac. surg. 2004;62:3-8.

8. Esen E, Tassar F, Akhan O. Determination of the anti-inflammatory effects of methylprednisolone on the sequelae of third molar surgery. J. oral maxilofac. surg. 1999; 57(10):1201-1206.

9. Marzola C. Técnica exodôntica. $3^{a}$ Ed. São Paulo: Pancast; 2000.

10. Waldron CA. Cistos e tumores odontogênicos. In: Neville BW, Damm DD, et al. Patologia oral e maxillofacial. $3^{a}$ Ed. Rio de Janeiro: Elsevier; 2009. p. 684-688.

11. Frederiksen NL. Técnicas especiais de imagem. In: White SC, Pharoah MJ. Radiologia Oral (Fundamentos e Interpretação). $5^{\mathrm{a}} \mathrm{Ed}$. Rio de Janeiro: Elsevier; 2007. p. 247-64.

12. Bouloux GF, Steed MB, Perciaccante VJ. Complications of third molar surgery. Oral maxillofac. surg. clin. North Am. 2007;19(1):117128.

13. Peixoto et al. Controle da dor pós-operatória em cirurgia oral: revisão da literatura. Rev. bras. ciênc. saúde. 2011;15(4):465-70.

14. Teixeira MJ. Fisiopatologia da Nocicepção e da supressão da dor. Jornal brasileiro de oclusão, ATM e dor orofacial. 2001;1(4):329-334.

15. Silva JA, Ribeiro-Filho NP. A dor como um problema psicofísico. Rev. dor. 2011; 12(2): 138151.

16. Wannmacher L, Ferreira MBC. Princípios gerais do correto tratamento da dor. In: Wannmacher L, Ferreira MBC. Farmacologia clínica para dentistas. $2^{\mathrm{a}}$ Ed. Rio de Janeiro: Editora Guanabara; 2007. p. 99-103.

17. Ministério da Saúde (BR). Cuidados paliativos oncológicos - controle da dor. Rio de Janeiro: INCA; 20O2. p. 328:13-117. (Manuais técnicos).

18. Bottega FH, Fontana RT. A dor como quinto sinal vital: utilização da escala de avaliação por enfermeiros de um hospital geral. Texto \& contexto enferm. 2010;19(2):283-290. 
19. Negreiros RM. Cirurgia de terceiros molares: avaliação da dor, edema, qualidade de vida e variações conforme posição dental [mestrado]. São Paulo: Universidade de São Paulo; 2010.

20. Aguiar ASW, Oliveira ACX, Martins PC, Freire ROM. Avaliação do grau de abertura bucal e dor pós-operatória após a remoção de terceiros molares inferiores retidos. Rev. cir. traumatol. buco-maxilo-fac. 2005;5(3):57-64.

21. Sortino F, Cicciù M. Strategies used to inhibit postoperative swelling following removal of impacted lower third molar. Dent. res. j. 2011;8(4):162-170.

22. Pell GJ, Gregory BT. Impacted mandibular third molars classification and modified technique for removal. Dental dig. 1933;39:330-8.

23. Silva JCL. Avaliação de dois protocolos em exodontias de terceiros molares inferiores retidos [mestrado]. Piracicaba: Universidade Estadual de Campinas, Faculdade de Odontologia de Piracicaba; 2011.

24. Saar JT, Leibur E, Tamme TO. Efeito da prednisolona na redução de reclamações depois da remoção cirúgica de terceiros molares impactados. Stomatologija. 2010;12:17-22.

25. Simone JL, Jorge WA, Horliana ACRT, Canaval TG, Tortamano IP. Comparative analysis of preemptive analgesic effect of dexamethasone and diclofenac following third molar surgery. Braz. oral res. 2013;27(3):266-71.
26. Murugesan; et al. Comparassion of the roles serratiopeptidase and dexametasone in the control of inflammation and trismus following impacted third molars surgery. Indian j. dent. res. 2012;23(6):709-716.

27. Leone M, Richard $O$, Antonini F, Rousseau $S$, Chabaane W, Guyot L, Martin C. Comparasion of methylprednisolone and ketoprofen after multiple third molar extraction: a randomized controlled study. Oral surg. oral med. oral pathol. oral radiol. endod. 2007; 103(1):7-9.

28. Koccer G, Yuce E, Tuzuner Oncul A, Dereci O, Koskan O. Effect of the route of administration of methylprednisolone on edema and trismus in impacted lower third molar surgery. J. oral maxillofac. surg. 2014;43:639-643.

29. Trindade PAK, Fernando PM, et al. Sublingual ketorolac and sublingual piroxicam are equally effective for postoperative pain, trismus and swelling management in lower third molar removal. Oral surg. oral med. oral pathol. oral radiol. endod. 2012;114(1):27-34.

30. Calvo $A M$ et al. Comparasion of the efficacy of etoricoxib and ibuprofen in pain and trismus control after lower third molar removal. Rev. odontol. Univ. São Paulo. 2006;18(1): 29-36.

31. Menezes SAF; Cury PR. Efficacy of nimeluside versus meloxican in the control of pain, swelling and trismus following extraction of impacted lower third molar. J. oral maxillofac. surg. 2010;39:580-4. 